\title{
Enhancing Measurement Quality through Active Sampling in Mobile Air Quality Monitoring Sensor Networks*
}

\author{
Adrian Arfire, Ali Marjovi and Alcherio Martinoli
}

\begin{abstract}
In recent years, a growing number of research groups have targeted the development and deployment of networks using low-cost chemical sensors for monitoring air quality. Due to economical reasoning, most of these systems make use of some sort of mobility to increase spatial coverage. The effect of mobility on measurement quality has, however, been largely neglected. The long response time of the chemical sensors typically used for this type of application, in conjunction with platform mobility, leads to significant signal distortion. While this problem can be addressed through signal deconvolution techniques, their effectiveness is limited by the typical poor Signal-to-Noise Ratio (SNR) of the measured signal. In this paper, we study the possibility of enhancing the measurement quality of chemical sensors through the use of active sampling (or sniffing). We propose different sniffer designs, employing both fans and pumps as actuators. Using a rigorous experimental framework, inside a wind tunnel, we study the ability of active samplers to increase measurement SNR, and thus indirectly to improve sensor dynamic response. We obtain a significant and consistent improvement in SNR for one of our pump-based sniffer designs. Finally, we validate the robustness of this signal enhancement in real-world conditions through an outdoor car-based experiment.
\end{abstract}

\section{INTRODUCTION}

Air pollution represents a major concern for human health, affecting both life expectancy and quality of life [1], and being singled out as the current number one environmental health risk by the World Health Organization [2].

The established method for long term air quality monitoring is the use of large static stations, grouped into national networks. These stations are equipped with highly accurate, but very expensive measurement equipment (e.g., absorption spectrophotometers, mass spectrometers, etc.). The high cost of these stations means that they form very sparse networks (e.g., the Swiss National Air Pollution Monitoring Network NABEL - uses a total of 16 stations for the whole country). This implies a very low spatial resolution of measurements, which limits the ability of capturing the spatial heterogeneity of the air pollution field.

Spatial heterogeneity of air pollution fields is particularly large in urban environments because of factors such as the locality of emission sources (e.g., industries, traffic patterns) and the specific urban landscape (e.g., topography, street canyons, green areas, etc.).

\footnotetext{
*This work was funded by Nano-Tera.ch (www.nano-tera.ch), a research initiative scientifically evaluated by the Swiss National Science Foundation and financed by the Swiss Confederation, in the framework of the OpenSense II project (http://opensense.epfl.ch).

The authors are with the Distributed Intelligent Systems and Algorithms Laboratory, School of Architecture, Civil and Environmental Engineering, École Polytechnique Fédérale de Lausanne (EPFL), Lausanne, Switzerland. E-mail addresses: firstname.lastnamedepfl.ch
}

\section{A. Mobile Air Quality Sensing}

Over the last decade, the field of Wireless Sensor Networks (WSNs) has seen a growing interest in the development and deployment of networks that employ small low-cost sensors for air quality monitoring. Since the cost of covering a whole city with a purely static WSN would still be impractically high, the number of projects that do not consider mobility is relatively small [3], [4], most projects considering either a mix of static and mobile nodes, or exclusively mobile deployments. Most urban mobility sources have been considered, including pedestrians [5]-[8], cyclists [9], [10], private vehicles [11], and public transportation [12]-[14]. Our work is part of the OpenSense II project, which falls into the latter mobility category by using buses in the city of Lausanne, and trams in Zurich. Nevertheless, the results presented in this paper are general and not limited to this type of mobility.

The main advantage of using mobility stems from the possibility of extending spatial coverage for a given number of sensor nodes. Nonetheless, the inherent dynamic nature of mobile coverage represents a significant challenge for attaining spatio-temporally complete high-resolution air pollution maps. This issue can be addressed through statistical modeling techniques, as suggested by Hasenfratz et al. [15], $\mathrm{Li}$ et al. [16], or Marjovi et al. [14]. However, all of these works have considered solely Particulate Matter (PM) measurements, and to the best of our knowledge, no similar results have been published for gaseous pollutants using mobile measurement data.

The reason is that the current commercially available chemical sensors for measuring gas-phase pollutants suffer from a number of issues that need to be addressed before applying the aforementioned modeling methods. These include temporal drift, due to sensor aging, cross-sensitivities to other chemical components or environmental parameters (e.g., temperature, humidity, pressure, etc.) [17], [18], low SNR at ambient concentration levels, and slow response times, which, in the context of mobility, leads to significant measurement distortion.

The problems of sensor drift and cross-sensitivity can be addressed through on-line calibration methods, and, although still an open problem, a significant body of work has been building up on this topic in recent years [19]-[23]. The issue of signal distortion due to the interplay of slow sensor response and high platform mobility has, however, received little attention.

In previous work [24], we showed that this is a significant problem and proposed mitigating it through 


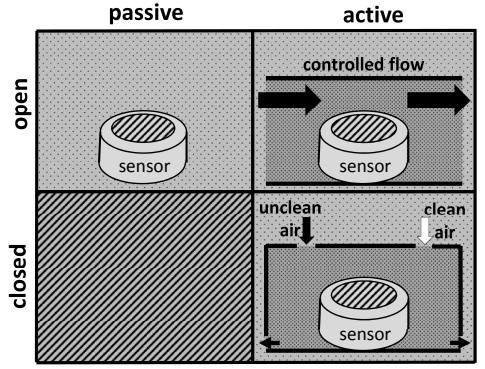

Fig. 1. Classification of sampling systems based on how the sensor is exposed to air. See text for descriptions of each class. Closed passive systems do not exist, as a sensor would be isolated from external air in such a setup.

signal deconvolution and the use of an active air sampling system (i.e. a sniffer). When reconstructing the signal through deconvolution, we were able to obtain consistent improvements in both feature localization accuracy (i.e. the ability to assign correctly the position of discrete features in the field), and Root Mean Square Error (RMSE), relative to the estimated ground-truth. The performance of the basic sniffer we considered was, however, very limited in terms of SNR enhancement.

The work presented in this paper comes to complement that through a sustained effort to obtain a sniffer design that would provide a consistent and robust enhancement of the raw measurement signal. Since the main limiting factor for the deconvolution technique we considered was due to falling SNR with increased sensing platform speed, a careful consideration of the sampler design is well motivated.

\section{B. Active Sampling}

According to the schematic representation in Fig. 1, air sampling systems can be broadly classified as either open passive, open active, or closed active systems.

Open passive systems rely only on existing background air flows for transporting the gas molecules to the sensitive surface of the sensor, and, due to their simplicity, are the cheapest and currently most widely spread type of samplers in mobile WSN applications [6]-[9], [11].

Open active systems, also called sniffers, use an actuator like a fan or a pump to draw and flush air around the sensor for improving its response. Measurement systems that can be classified in this class have been previously used in a limited number of mobile WSN projects [12], [15], [25], but no work on the analysis of their design or their effectiveness has been published to date. The design of sniffers has been studied more in the field of robotic olfaction, with published work including both fan-based [26] and pump-based systems [27], [28]. However, these designs were developed for indoor applications, where background flows are very limited compared to outdoor conditions.

Finally, closed active systems, also known as closed chamber systems, are the most complex air sampling systems. They have a typical three-phase measurement cycle. Firstly, an air sample is pumped into a chamber containing the sensors. Secondly, the pump is turned off while the sensor

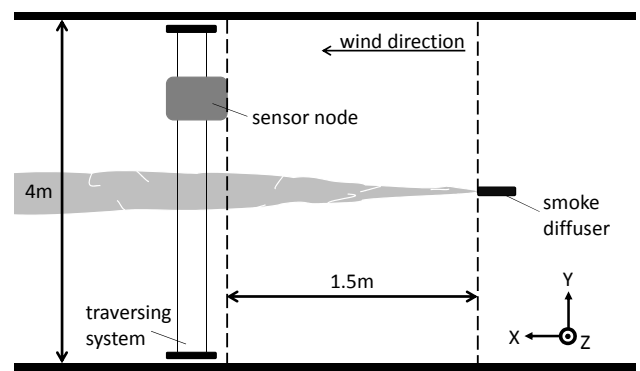

Fig. 2. Top view of experimental setup inside wind tunnel (not to scale).

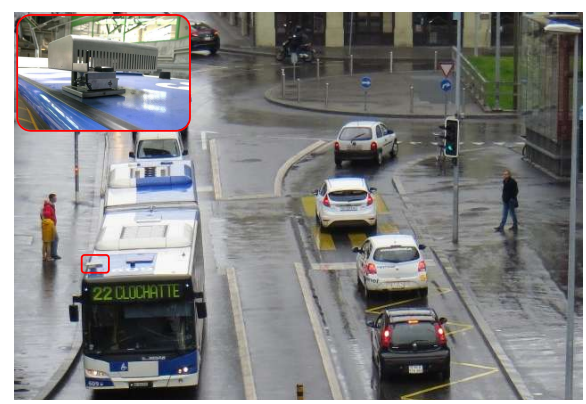

Fig. 3. One of our sensor nodes anchored to the roof of a Lausanne bus. The gas sampling system used in this paper is a sub-module of this real-world platform.

is allowed the time to reach a stable reading. Finally, the air sample is flushed out of the chamber while allowing the sensor to recover its baseline, typically using an additional source of clean air. While this type of system has the unique advantage of permitting absolute concentration measurements, its complexity and inherent low-sampling rate make it less attractive for mobile monitoring applications.

\section{Our Contribution}

In this paper, we study the opportunity of using open active samplers for enhancing the quality of chemical sensor measurements in mobile applications. We consider both fanand pump-based designs and study their ability to improve the measured signal in terms of SNR.

We use a rigorous wind tunnel experimental framework for the performance evaluation of the considered design options. For the best performing sniffer, we go a step forward and investigate if its performance is maintained when moving outside of the wind tunnel, in a real-world comparative experiment using an electric car as mobility source.

\section{EXPERIMENTAL SETUP}

We conducted most of our experiments in a controlled environment - a boundary layer wind tunnel. The only exception was one comparative outdoor experiment using an electric car, which is described in detail in Section V.

The experimental setup is similar to the one we used in [24] and is represented schematically in Fig. 2. The gas sampling system we used is a sub-module of the mobile air quality sensing system we developed and deployed on 10 buses in the city of Lausanne within the OpenSense and OpenSense II 

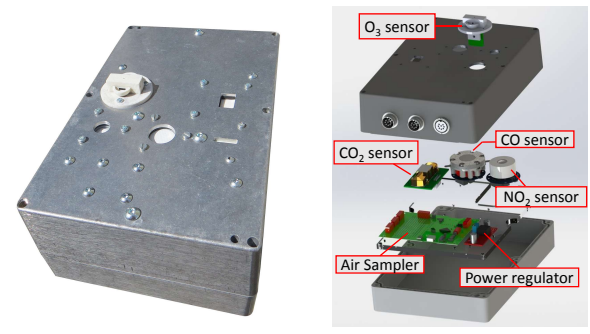

Fig. 4. Closed (left) and exploded (right) views of the gas sampler box. In its standard form it is an open passive sampler.

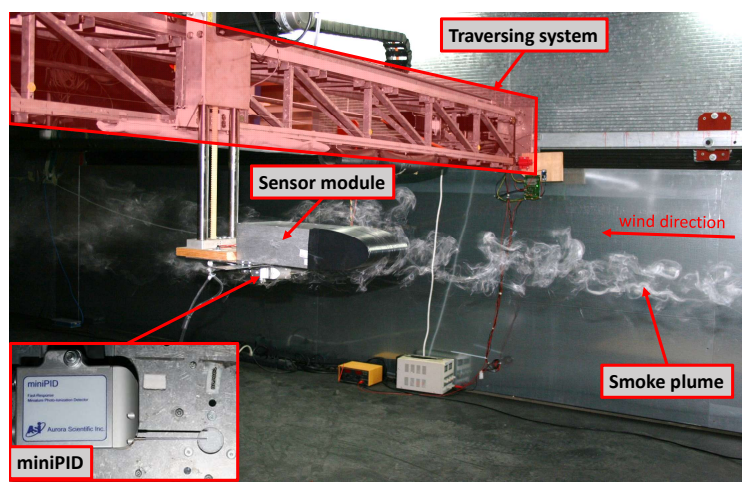

Fig. 5. Sensor node traversing the smoke plume inside our wind tunnel.

projects (see Fig. 3 and Fig. 4). Due to its selectivity, we continued to focus only on the City Technology A3CO carbon monoxide electrochemical sensor [29].

The experiments were performed in a $19.5 \times 4 \times 1.95 \mathrm{~m}^{3}$ test section of our wind tunnel. We anchored the sensor box to the tunnel's traversing system, a 3-axis cartesian robot, with the sensors facing down, as they are also in our bus deployment. We generated a chemical plume by using the Pea Soup Wind Tunnel Air Flow Tracer SGS-90 [30] smoke machine mounted at a height of $45 \mathrm{~cm}$ from the floor.

Differently from [24], as ground truth we used the fast response 200B miniPID measurement system [31], a photoionization detector, which we attached in close vicinity of our electrochemical sensor. Both the miniPID and the electrochemical sensor are sensitive to the plume generated by the smoke machine. A snapshot of our experimental setup, including the positioning of the miniPID, is shown in Fig. 5.

\section{Active SAmpler Designs}

In this section, we present the active sampler designs we considered in our experiments. An open active sampler (or open sniffer) is an air sampling system that uses an actuator to enhance the air flow around a chemical sensor, with the goal of improving its response when compared to a purely passive system. The actuator can be either a fan or a pump, and in this work we consider both cases.

We guided our design effort by using Computational Fluid Dynamics (CFD) simulations, in order to understand the impact of our different design choices on the air flow, before moving to experimental trials. With this approach, we first
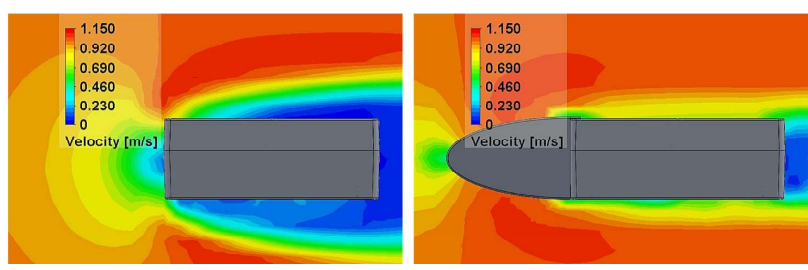

Fig. 6. Sensor box without (left) and with (right) aerodynamic profile.
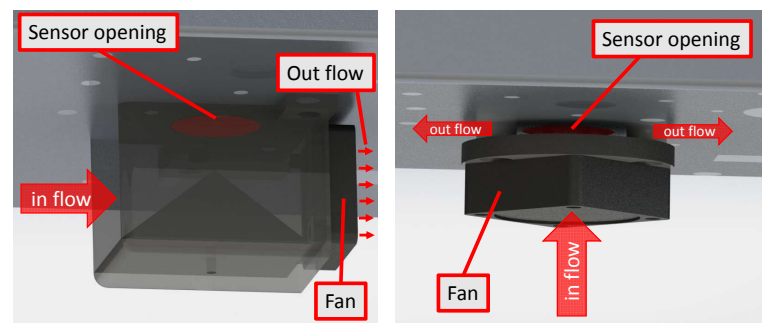

Fig. 7. Lateral flow (left) and normal flow (right) fan-based sniffers.

considered how the air flow around the sensor box could be enhanced through a purely passive solution: modifying the aerodynamic profile of the node enclosure. As can be seen in Fig. 6, adding an elliptical profile to the front of the sensor box reduces significantly the thickness of the boundary layer around the sensor box. The choice of this particular profile shape was influenced by the elliptical conical noses used in sub-sonic flying platforms. All experiments presented in this paper were done using a configuration that includes this aerodynamic profile.

\section{A. Fan-based sniffer designs}

The fan-based sniffers we considered employ axial fans to produce air flow. Axial fans force the air to move parallel to their rotating shaft and are capable to move large volumes of air. However, their performance can be significantly influenced by the background flow conditions.

We considered two types of fan-based sniffers (see Fig. 7):

- A lateral flow sniffer has the design we previously used in [24], with a fan mounted at the end of an enclosure which pulls the air over the sensor, and contains a wedge to direct flow towards the sensor.

- A normal flow sniffer design represents an axial fan mounted with its shaft perpendicular to the sensing surface through a supporting structure. In this case the air is pushed directly towards the sensor.

Multiple axial fans were tested on these designs with flow rates ranging from $3.74 \mathrm{~m}^{3} / \mathrm{h}$ to $33 \mathrm{~m}^{3} / \mathrm{h}$.

\section{B. Pump-based sniffer designs}

For the pump-based designs we used the $0.22 \mathrm{~m}^{3} / \mathrm{h}$ diaphragm pump (Sensidyne AP240DEEE Model 60 [32]). This type of device is a positive displacement pump, having the advantage of ensuring an almost constant air flow, being less susceptible to environment conditions. Nonetheless, the volume of air that diaphragm pumps can move is small relative to their size. 


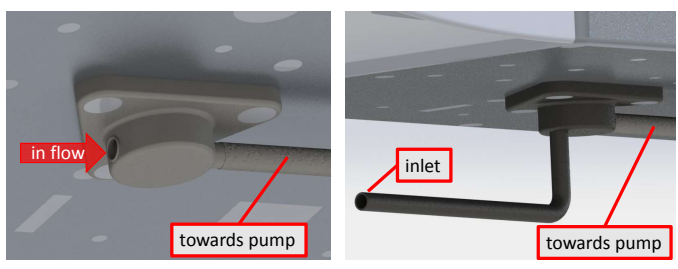

Fig. 8. Leveled-inlet (left) and raised-inlet (right) pump-based sniffers. In both cases the air is pulled through a narrow channel towards the sensor surface and is then evacuated through the pump out through an small opening at the back of the sensor box.

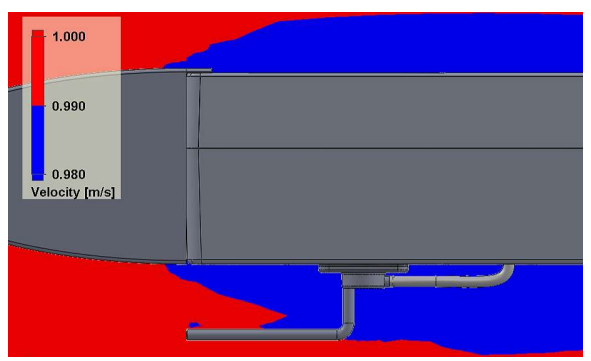

Fig. 9. A clearance of $3 \mathrm{~cm}$ from the box surface ensures that the inlet escapes the boundary layer (in blue above). The simulation in this figure was performed for a background air flow of $1 \mathrm{~m} / \mathrm{s}$.

In this case also, we considered two design options for our active sampler (see Fig. 8):

- A leveled-inlet sniffer is a basic pump-based design similar to the one used by Lochmatter et al. in [28], and has the inlet opening in close proximity of the sensor box surface.

- A raised-inlet sniffer is a modification of the above design, in which the inlet is extended and raised outside of the boundary layer formed around the sensor box.

In order to choose the distance from the box surface, that the inlet needs to be raised to, we used CFD simulations and the definition of the boundary layer thickness, as being the level at which the air velocity is equal to $99 \%$ of the free flow velocity (see Fig. 9). We, thus, found that a clearance of $3 \mathrm{~cm}$ from the sensor box surface is sufficient to place the inlet outside of the boundary layer.

\section{RESUlts}

We are interested in enhancing the measured signal from a SNR perspective, and thus indirectly providing better conditions for compensating the slow sensor dynamic response through appropriate signal processing techniques.

We use the common definition of the SNR as the ratio between the power of the a signal and the power of the background noise. In our scenario, the separation between noise and signal is done based on the assumption that the signal is null outside of the smoke plume (i.e. a direct measurement of sensor noise).

In order to estimate the sensor rise time, we use Matlab's System Identification Toolbox to determine the parameters of a time-delayed over-damped second-order linear sensor model with a transfer function in the frequency domain of

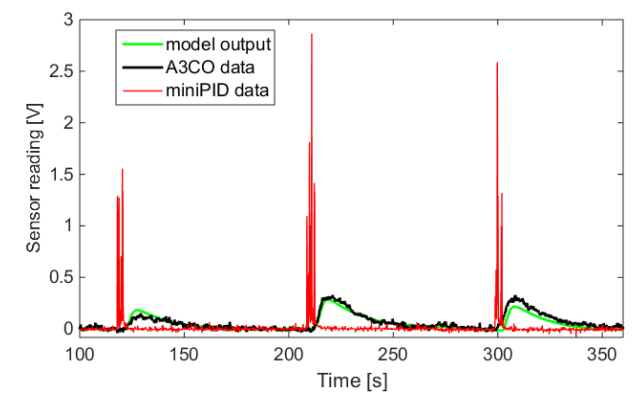

Fig. 10. Partial view of system identification data-set. A complete set uses 10 experiments.

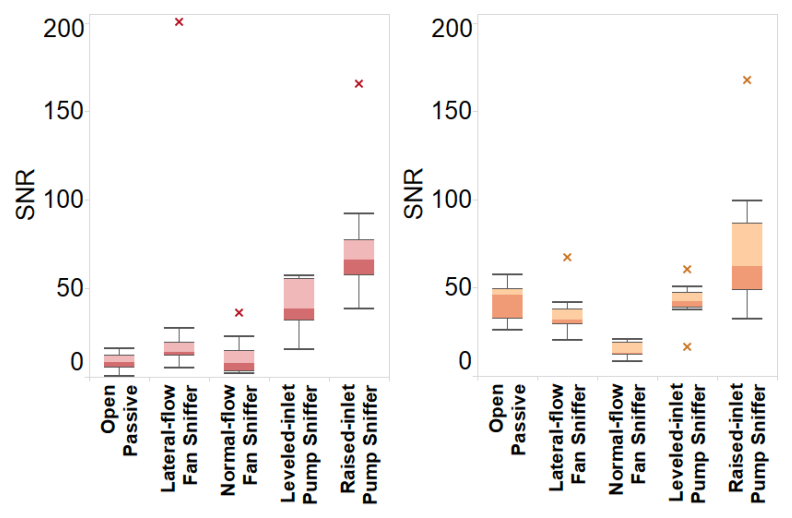

Fig. 11. Comparison of sampler system performance at a height of $49 \mathrm{~cm}$ (left), and $51 \mathrm{~cm}$ (right).

the form:

$$
H(s)=e^{-t_{d} s} \frac{K}{\left(s+c_{0}\right)\left(s+c_{1}\right)}
$$

where $K$ is a constant parameter of the model, and $c_{0}$ and $c_{1}$ are its two poles. The time-delay $\left(t_{d}\right)$ is a refinement of the sensor model we used in [24], which provides a better fit to the experimental data. In the identification process we use the miniPID data as input and the electrochemical sensor data as system output. Fig. 10 shows an example of an identification input-output data-set pair, and the identified model output.

We performed sets of ten experiments for each sampler system configuration, traversing speed, and wind speed we considered. Throughout all our experiments both the electrochemical sensor and the miniPID were sampled at $10 \mathrm{~Hz}$. We performed movements at constant speed on the Y-axis in increments starting from $5 \mathrm{~cm} / \mathrm{s}$ and up to $30 \mathrm{~cm} / \mathrm{s}$. However, due to the narrow width of the plume (approx. $20 \mathrm{~cm}$ ), most of the experiments were performed at the lower end of this range.

Other parameters that we systematically varied are the wind speed, from $0.97 \mathrm{~m} / \mathrm{s}$ up to $2.62 \mathrm{~m} / \mathrm{s}$, and the level on the $\mathrm{Z}$-axis of the traversing system. We performed in total more than eight hundred single experiments. The results presented in this section are the most significant we obtained.

Fig. 11 presents the performance in terms of SNR obtained with our sampling systems for different coordinates on the $\mathrm{Z}$-axis of the traversing system. The traversing speed for these experiments was $5 \mathrm{~cm} / \mathrm{s}$, and the wind speed was $0.97 \mathrm{~m} / \mathrm{s}$. 


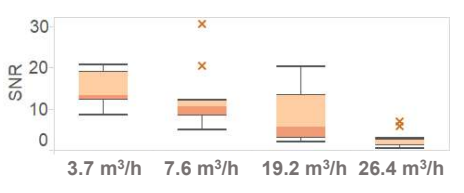

Fig. 12. The SNR of the normal flow fan-based system designs drops with the increase of the nominal fan flow. These experiments were performed at a height of $51 \mathrm{~cm}$.

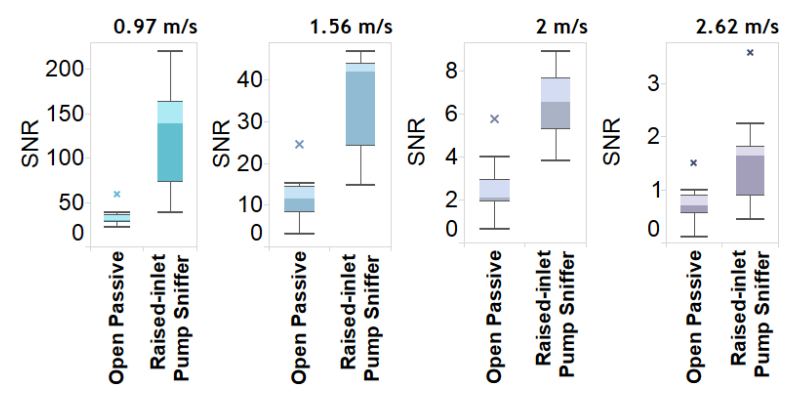

Fig. 13. The performance of the raised-inlet pump-based sniffer is robust to large variations of the wind speed. These experiments were performed at the same level as the smoke machine (i.e. $45 \mathrm{~cm}$ from the floor).

As can be seen, the pump-based sniffers perform better than the fan-based sniffers. The leveled-inlet sniffer performed at least as well as the passive sampler, if not better, while the raised-inlet sniffer was consistently the best of all the sampling systems considered, irrespective of the considered traversing height.

The fan-based sniffers, however, illustrate an inconsistent performance - outperforming the open passive sampler for a certain traversing height, but performing worse than it for another. It should be noted that the presented results for the fan-based sniffers consider only the configuration with the best performing actuator, which for both types of sniffers was the small $3.74 \mathrm{~m}^{3} / \mathrm{h}$ Delta AFB02512HH [33]. This was the least powerful fan considered in our study.

In fact, for the normal-flow sniffer design, we were able to observe a monotonously decreasing relationship between the performance of the sniffer and the nominal airflow of the fans we considered (see Fig. 12). This is probably due to a mix between re-circulation of air around the fan-supporting platform, and the destructive effect of the fan on the plume structure, which we could actually observe visually.

In order to further test the robustness of the SNR enhancement by the raised-inlet pump sniffer, we performed experiments in which we incrementally varied the wind speed inside the channel, and compared the performance of the open passive and active samplers. The results in Fig. 13 show that, while there is a general decrease in the SNR with increasing wind speeds (due to the narrowing of the smoke plume), the raised-inlet pump-based sniffer consistently outperforms the open passive sampler.

While we were able to have an effect on the SNR with our different sampler designs, it is worth noting that there was no observable effect on the sensor dynamics, with the estimated values for the rise time being clustered around

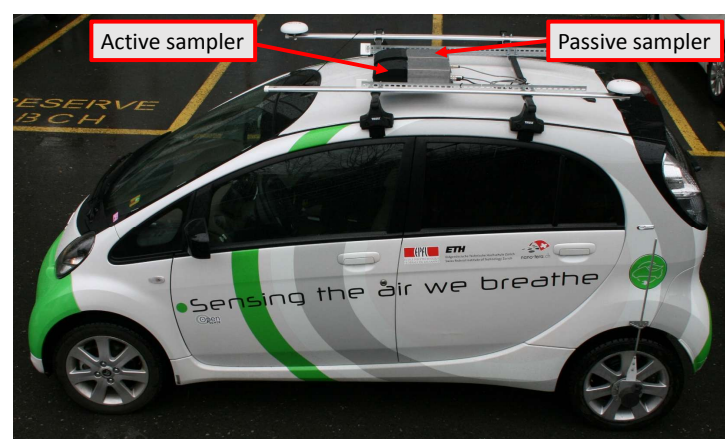

Fig. 14. Electrical car used for our outdoor experiment.

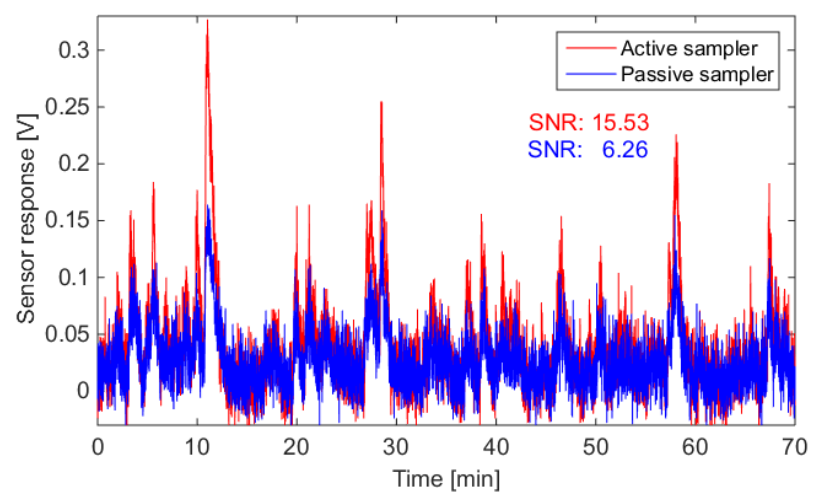

Fig. 15. The results of the real-world experiment confirms the benefit of using the active sampler for enhancing the SNR.

the value of 29.2 seconds, with a standard deviation of 2.9 seconds, in line with the manufacturer's specifications (i.e. less than 40 seconds).

\section{OUtDOor ExPERIMENTAL VALIDATION}

After concluding that the raised-inlet pump-based sniffer provided the best performance in our controlled experiments, we decided to test it in an uncontrolled outdoors experiment. To this end, we equipped an electric car (see Fig. 14) with two gas sensor boxes with similar SNR characteristics evaluated inside the wind tunnel.

One of the two sensor boxes was equipped with a raisedinlet pump-based sniffer, while the other one was left as a passive sampler. The measurements were geo-tagged and recorded using the localization module and data-logger developed for the Lausanne deployment of the OpenSense and OpenSense II projects (i.e. the same type of system we use on buses).

We performed an experiment of more than one hour, driving from the outskirts of the EPFL campus to the center of Lausanne where we performed ten loops in an area with regular traffic, and then returned to EPFL. The results of this experiment are shown in Fig. 15. As can be seen from this graph, the two measured signals are very well correlated, but the peaks of the sensor box using our sniffer are generally higher in amplitude. The SNR values calculated for the two data-sets confirm a very robust enhancement of the raw measurement signal provided by the active sampler. 


\section{CONCLUSIONS}

We investigated the potential of active samplers to enhance the quality of chemical sensor readings in mobile applications for air quality monitoring. We performed rigorous repeatable experiments in a controlled environment, a wind tunnel, to study the benefit of using sniffers for this type of application.

We considered both fan- and pump-based sniffer designs and found that the latter are generally better suited as a sniffing actuator. In particular, one of our proposed pumpbased designs, which places the inlet opening outside of the boundary layer formed around the sensor box, delivered consistently superior results in SNR enhancement compared to the baseline passive sampler. This result was confirmed also outside the controlled environment of the wind tunnel, through a real-world vehicle-based experiment that we conducted using two sensor boxes in parallel.

We conclude that mobile sensing systems for air quality monitoring can indeed benefit in terms of SNR enhancement from the use of well-designed active samplers. Although in this work we focused solely on enhancing the raw measurement signal, the use of active sampling is complementary to the deconvolution method we presented in [24], as an increase in SNR will improve signal reconstruction.

\section{REFERENCES}

[1] R. Beelen, O. Raaschou-Nielsen, M. Stafoggia, Z. J. Andersen, G. Weinmayr, B. Hoffmann, K. Wolf, E. Samoli, P. Fischer, and M. Nieuwenhuijsen, "Effects of long-term exposure to air pollution on natural-cause mortality: an analysis of 22 european cohorts within the multicentre escape project," The Lancet, vol. 383, no. 9919, pp. 785-795, 2014.

[2] World Health Organization (WHO). News release, 25 March 2014, Geneva. http://www.who.int/mediacentre/news/releases/2014/ air-pollution/en/. [Online; accessed 5 March 2016].

[3] Centre for Scientific Computing, University of Cambridge. Sensor Networks for Air Quality at Heathrow Airport. http://www.snaq.org/. [Online; accessed 25 September 2015].

[4] Y. Cheng, X. Li, Z. Li, S. Jiang, Y. Li, J. Jia, and X. Jiang, "Aircloud: a cloud-based air-quality monitoring system for everyone," in Proceedings of the 12th ACM Conference on Embedded Network Sensor Systems, 2014, pp. 251-265.

[5] Imperial College London. MESSAGE - Mobile Environmental Sensing System Across Grid Environments. http://www3.imperial.ac.uk/lesc/ projects/archived/mobileenviron. [Online; accessed 25 September 2015].

[6] E. Bales, N. Nikzad, N. Quick, C. Ziftci, K. Patrick, and W. Griswold, "Citisense: Mobile air quality sensing for individuals and communities design and deployment of the citisense mobile air-quality system," in The 6th International Conference on Pervasive Computing Technologies for Healthcare, 2012, pp. 155-158.

[7] Preemptive Media. AIR - Area's Immediate Reading. http://www.pm-air. net/index.php. [Online; accessed 25 September 2015].

[8] P. Dutta, P. M. Aoki, N. Kumar, A. Mainwaring, C. Myers, W. Willett, and A. Woodruff, "Common sense: participatory urban sensing using a network of handheld air quality monitors," in Proceedings of the 7th ACM conference on embedded networked sensor systems, 2009, pp. 349-350.

[9] SENSEable City Laboratory, MIT. The Copenhagen Wheel. http:// senseable.mit.edu/copenhagenwheel/. [Online; accessed 25 September 2015].

[10] B. Elen, J. Peters, M. V. Poppel, N. Bleux, J. Theunis, M. Reggente, and A. Standaert, "The aeroflex: a bicycle for mobile air quality measurements," Sensors, vol. 13, no. 1, pp. 221-240, 2012.

[11] W. Hedgecock, P. Völgyesi, A. Ledeczi, X. Koutsoukos, A. Aldroubi, A. Szalay, and A. Terzis, "Mobile air pollution monitoring network," in Proceedings of the 2010 ACM Symposium on Applied Computing, 2010, pp. 795-796.
[12] N. Castell, H.-Y. Liu, M. Kobernus, A. J. Berre, J. Noll, E. Cagatay, and R. Gangdal, "Mobile technologies and personalized environmental information for supporting sustainable mobility in Oslo: The citi-sensemob approach," in 28th EnviroInfo Conference, 2014, pp. 699-706.

[13] D. Hasenfratz, O. Saukh, and L. Thiele, "Model-driven accuracy bounds for noisy sensor readings," in 9th International Conference on Distributed Computing in Sensor Systems, 2013, pp. 165-174.

[14] A. Marjovi, A. Arfire, and A. Martinoli, "High resolution air pollution maps in urban environments using mobile sensor networks," in 11th International Conference on Distributed Computing in Sensor Systems, 2015, pp. 11-20.

[15] D. Hasenfratz, O. Saukh, C. Walser, C. Hueglin, M. Fierz, T. Arn, J. Beutel, and L. Thiele, "Deriving high-resolution urban air pollution maps using mobile sensor nodes," Pervasive and Mobile Computing, vol. 16, pp. 268-285, 2015.

[16] J. J. Li, A. Jutzeler, and B. Faltings, "Estimating urban ultrafine particle distributions with gaussian process models," In S. Winter and C. Rizos (Eds.): Research@Locate14,pp. 145-153, 2014.

[17] M. Aleixandre and M. Gerboles, "Review of small commercial sensors for indicative monitoring of ambient gas," Chemical Engineering Transactions, vol. 30, pp. 169-174, 2012.

[18] E. G. Snyder, T. H. Watkins, P. A. Solomon, E. D. Thoma, R. W. Williams, G. S. Hagler, D. Shelow, D. A. Hindin, V. J. Kilaru, and P. W. Preuss, "The changing paradigm of air pollution monitoring," Environmental Science \& Technology, vol. 47, no. 20, pp. $11369-$ $11377,2013$.

[19] D. Hasenfratz, O. Saukh, and L. Thiele, "On-the-fly calibration of low-cost gas sensors," Wireless Sensor Networks, pp. 228-244, 2012.

[20] O. Saukh, D. Hasenfratz, C. Walser, and L. Thiele, "On rendezvous in mobile sensing networks," in Real-World Wireless Sensor Networks, 2014, pp. 29-42.

[21] O. Saukh, D. Hasenfratz, and L. Thiele, "Reducing multi-hop calibration errors in large-scale mobile sensor networks," in 14th International Conference on Information Processing in Sensor Networks, Seattle, WA, USA, Apr 2015, pp. 274-285.

[22] A. Arfire, A. Marjovi, and A. Martinoli, "Model-based rendezvous calibration of mobile sensor networks for monitoring air quality," in Proceedings of the IEEE Sensors Conference 2015, 2015, pp. 366-369.

[23] B. Maag, O. Saukh, D. Hasenfratz, and L. Thiele, "Pre-deployment testing, augmentation and calibration of cross-sensitive sensors," in International Conference on Embedded Wireless Systems and Networks, 2016, pp. 169-180.

[24] A. Arfire, A. Marjovi, and A. Martinoli, "Mitigating slow dynamics of low-cost chemical sensors for mobile air quality monitoring sensor networks," in International Conference on Embedded Wireless Systems and Networks, 2016, pp. 159-167.

[25] B. Elen, J. Theunis, S. Ingarra, A. Molino, J. Van den Bossche, M. Reggente, and V. Loreto, "The everyaware sensorbox: a tool for community-based air quality monitoring," in Sensing a Changing World Workshop, 2012.

[26] A. Lilienthal and T. Duckett, "A stereo electronic nose for a mobile inspection robot," in 1st International Workshop on Robotic Sensing, 2003.

[27] A. Kohnotoh and H. Ishida, "Active stereo olfactory sensing system for localization of gas/odor source," in 7th International Conference on Machine Learning and Applications, 2008, pp. 476-481.

[28] T. Lochmatter and A. Martinoli, "Tracking an odor plume in a laminar wind field with the crosswind-surge algorithm," in Proc. of the Eleventh Int. Symp. Experimental Robotics, vol. 54, 2008, pp. 473-482.

[29] City Technology Ltd. A3CO Sensor Datasheet. https://www.citytech. com/PDF-Datasheets/a3co.pdf. [Online; accessed 26 September 2015].

[30] Pea Soup Ltd. Wind Tunnel Air Flow Tracer Brochure. http:// www.smokemachines.net/PDF/Air-flow-tracer-brochure.pdf. [Online; accessed 26 September 2015].

[31] Aurora Scientific Inc. 200B miniPID Fast Response Gas Sensor. http: //hubspot.aurorascientific.com/product/fr-minipid/. [Online; accessed 5 March 2016].

[32] Sensidyne, Inc. A Plus DEEE All Specifications. http: //www.sensidyne.com/library/micro-air-pumps/A\%20Plus\% 20DEEE\%20All\%20Specs.pdf. [Online; accessed 29 May 2016].

[33] Delta Electronics, Inc. AFB25x10mm-A Specifications. http://partner.delta-corp.com/Products/FANUploads/Specification/ AFB25x10mm-A.pdf. [Online; accessed 29 May 2016]. 